\title{
Polish validation of the Brief International Cognitive Assessment for Multiple Sclerosis (BICAMS battery): correlation of cognitive impairment with mood disorders and fatigue
}

\author{
Ewa Betscher ${ }^{1}$, Wojciech Guenter ${ }^{1,2}$, Dawn W. Langdon ${ }^{3}$, Robert Bonek ${ }^{1,4}$ \\ ${ }^{1}$ Department of Neurology and Clinical Neuroimmunology, Regional Specialist Hospital, Grudziadz, Poland \\ ${ }^{2}$ Chair and Department of Clinical Neuropsychology, Nicolaus Copernicus University, Torun, Poland; \\ Collegium Medicum, Bydgoszcz, Poland \\ ${ }^{3}$ Royal Holloway University of London, London, United Kingdom \\ ${ }^{4}$ Foundation Supporting Development of Neurology and Clinical Neuroimmunology MoA, Bydgoszcz, Poland
}

\begin{abstract}
Background: Cognitive impairment is recognised as a significant clinical issue in Multiple Sclerosis (MS). It can occur at any stage of the disease, affecting quality of life, occupational activity, and adherence to therapy. This makes the availability of a validated assessment tool for detecting and monitoring cognitive dysfunction in multiple sclerosis essential.

The Brief International Cognitive Assessment for Multiple Sclerosis is a practical and simple means of administering a battery of three neuropsychological tests, and does not require any formal neuropsychological training.

Objective: To establish the validity of BICAMS in the Polish MS population; to assess the correlations of cognitive status with demographic and clinical factors, including affective symptoms and fatigue.

Methods: BICAMS was administered to 61 MS patients and 61 HC subjects. Examination of 20 participants with MS was repeated after one to three weeks to assess test-retest reliability. The patients with MS and $\mathrm{HC}$ subjects also completed the Hospital Anxiety and Depression Scale (HADS) and Modified Fatigue Impact Scale (MFIS).

Results: The MS group performed worse than the HC group in all three BICAMS components, obtaining the following values respectively: 51.7 and $56.1(p=0.02)$ for CVLT, 25 and $28(p=0.03)$ for BVMT-R, and 48.8 and $57.2(p<0.001)$ for SDMT. All BICAMS tests had very significant correlations in test-retest reliability $(r=0.83, p<0.001$ for CVLT; $r=0.84, p<0.001$ for BVMTR; $r=0.9$, $p<0.001$ for SDMT). 34\% of MS patients presented cognitive dysfunction based on the criterion of one or more test scores below the 5 th percentile value of the HC group. Significant anxiety and depressive symptoms were reported by $31.1 \%$ and $18.0 \%$ of MS patients. 31.1\% of PwMS reported significant fatigue. BICAMS test results were not associated with HADS or MFIS scores. Conclusions: The Polish version of BICAMS is a valid and reliable tool for the assessment of cognitive impairment in patients with MS.
\end{abstract}

Key words: multiple sclerosis, cognitive impairment, bicams, validation

(Neurol Neurochir Pol 2021; 55 (1): 59-66)

Address for correspondence: Robert Bonek, Department of Neurology and Clinical Neuroimmunology, Regional Specialist Hospital, Grudziadz, Poland, e-mail: bonek.robert@gmail.com 


\section{Introduction}

Multiple Sclerosis (MS) is a chronic, inflammatory, autoimmune, and neurodegenerative disease of the central nervous system (CNS), characterised by the presence of demyelinating lesions disseminated in space and time. It occurs primarily in young adults. MS is a heterogeneous disease with differentiated clinical courses [1]. MS leads to a wide range of symptoms. Cognitive impairment (CI) is one of the most common clinical signs, occurring in 43-70\% of PwMS (patients with MS) [2-3]. CI has a negative influence on quality of life, resulting in occupational disability, as well as restrictions on participating in social activities and personal relationships [4].

Cognitive deficits occur at all stages of the disease and clinical courses. Patients with radiologically isolated syndrome (RIS) demonstrate signs of cognitive impairment. 18-57\% of patients have cognitive impairments after clinically isolated syndrome (CIS). $40 \%$ of patients with newly diagnosed MS have cognitive deficits [5-6]. The presence of $\mathrm{CI}$ in patients with CIS and RIS is associated with an increased risk of conversion to MS [7], and cognitive impairment at MS diagnosis is associated with a faster accumulation of physical disability [8].

The early diagnosis of cognitive dysfunction is therefore highly significant. In some patients $\mathrm{CI}$ may be the predominant, or even exclusive, sign in the clinical manifestation of MS [9].

Cognitive impairment is the most marked in secondary progressive multiple sclerosis (SPMS) and primary progressive multiple sclerosis (PPMS), with typically a more severe course in SPMS. This may be because of a longer disease duration in SPMS [10]. However, cognitive deterioration in PwMS is less severe than that noted in dementing neurological disorders such as Alzheimer's Disease. Cognitive processes in PwMS are often selectively affected. The most affected domains include information processing speed, attention, executive function, memory, and visuospatial abilities [3].

The relationship of cognitive deterioration to affective symptoms, as well as to fatigue, is complex. Mood disorders are common in PwMS. The most common is depression, which affects up to $50 \%$ of patients [11], while anxiety affects up to $40 \%$ of PwMS [12]. Patients with affective symptoms more often report cognitive disturbances but may not demonstrate objective cognitive impairment [13]. Subjective cognitive dysfunction is more highly correlated with depressive symptoms than with objective cognitive deterioration [14-15]. An association between $\mathrm{CI}$ and depression is evident in patients with severe symptoms of depression. However, this relation is not unidirectional [16]. The association between anxiety and $\mathrm{CI}$ is less well understood. However, some studies have indicated that anxiety may also impact upon cognitive performance, including information processing speed and executive function [17].

Fatigue is very common in PwMS, affecting up to $83 \%$ of individuals [18]. A correlation between fatigue and cognitive performance has been noted in some studies, especially in tasks demanding attention [19]. However, most studies have reported a lack of independent association between fatigue and cognitive performance $[15,20]$.

The poorly understood interrelations between cognitive dysfunction, affective symptoms, and fatigue complicate cognitive assessment in PwMS. Both an assessment of depressive symptoms and of fatigue should be included during cognitive function examination [13-14].

As cognitive dysfunction in MS can be focal and mild, a precisely targeted approach in neuropsychological assessment is necessary. Neuropsychological examination allows the detection of subtle cognitive disturbances, but has some limitations. There are resource implications for cognitive assessment. Various batteries of neuropsychological tests are recommended for PwMS. The most used are the Brief Repeatable Battery of Neuropsychological test (BRB-N) [21] and the Minimal Assessment of Cognitive Function in MS (MACFIMS) [22]. These batteries are time-consuming (administration takes 45 and 90 minutes respectively), and expensive, and need to be administered by specialised staff.

In 2012, an expert panel recommended the Brief International Cognitive Assessment in Multiple Sclerosis (BICAMS) [23], which is relatively short, simple to administer, and does not require specialised staff. This set consists of three tools considered sensitive in identifying cognitive dysfunction in PwMS including the Rao adaptation [21] of the Symbol Digit Modalities Test (SDMT) [24], the initial learning trials of the second edition of the California Verbal Learning Test (CVLT-II) [25] and the initial learning trials of the revised Brief Visuospatial Memory Test (BVMT-R) [26]. International standards for BICAMS validation have been developed to facilitate validation and use in many countries [27].

\section{Objectives}

The aims of this study were to:

1. Establish the validity of BICAMS in the Polish MS population

2. Assess the cognitive status of Polish PwMS using BICAMS

3. Assess the correlations of cognitive status with demographic and clinical factors, including affective symptoms and fatigue.

\section{Methods}

\section{Subjects}

A total of 61 patients aged 18 or over with Multiple Sclerosis with a diagnosis based on the revised McDonald criteria [28], were included in the study. Patients were treated at the Department of Neurology and Clinical Neuroimmunology of the Regional Specialist Hospital in Grudziadz, Poland. Patients were recruited cross-sectionally, and no selection for 
Table 1. Demographic characteristics in MS and HC groups

\begin{tabular}{lccc|} 
& $\begin{array}{c}\text { MS } \\
\mathbf{N = 6 1}\end{array}$ & $\begin{array}{c}\text { HC } \\
\mathbf{N = 6 1}\end{array}$ & $\mathbf{p}$ \\
\hline Age (years), median & 39 & 37 & 0.99 \\
(25-75 percentile) & $(28-49)$ & $(29-50)$ & \\
Education (years), median & 13 & 13 & 0.92 \\
(25-75 percentile) & $(12-17)$ & $(12-17)$ & \\
Gender (female/male), N (\%) & $45(74 \%) /$ & $46(75 \%) /$ & 0.84 \\
& $16(26 \%)$ & $15(25 \%)$ & \\
Employment status (employ- & $51(84 \%) /$ & $60(98 \%) /$ & 0.004 \\
ed/unemployed), N (\%) & $10(\mathbf{1 6 \% )}$ & $\mathbf{1 ( 2 \% )}$ &
\end{tabular}

Mann-Whitney U Test was used to assess the differences between the groups for age and education, $x^{2}$ was used for gender and employment status. MS - multiple sclerosis, HC — healthy control, significant differences $(p<0.05)$ marked in bold

Table 2. Demographic and clinical characteristics in MS subgroups

\begin{tabular}{lccc} 
& $\begin{array}{c}\text { RRMS } \\
\text { N = 45 }\end{array}$ & $\begin{array}{c}\text { SPMS } \\
\text { N=12 }\end{array}$ & $\begin{array}{c}\text { PPMS } \\
\mathbf{N}=\mathbf{4}\end{array}$ \\
\hline $\begin{array}{lccc}\text { Age (years), median } \\
\text { (25-75 percentile) }\end{array}$ & 33 & 47 & 57.5 \\
Gender (female/male), & $33(73.3 \%) /$ & $9(75 \%) /$ & $3(75 \%) /$ \\
N (\%) & $/ 12(26.7 \%)$ & $3(25 \%)$ & $1(25 \%)$ \\
Education (years), median & 15 & 12 & 12.5 \\
(25-75 percentile) & $(12-17)$ & $(11.5-17)$ & $(10-15)$ \\
Duration of disease (years), & 5 & 19.5 & 7.5 \\
median (25-75 percentile) & $(2.5-9)$ & $(15-25.5)$ & $(4.5-10)$ \\
EDSS, median & 3 & 4.75 & 4.5 \\
(25-75 percentile) & $(2-4)$ & $(4.25-6.5)$ & $(3.25-5.5)$
\end{tabular}

RRMS - relapsing-remitting multiple sclerosis, SPMS - secondary progressive multiple sclerosis, PPMS - primary progressive multiple sclerosis, EDSS — Expanded Disability Status Scale

cognitive impairment was performed before enrollment. The first language of all patients was Polish.

Exclusion criteria were:

- evidence of other neurological, psychiatric or systemic disease affecting cognitive function

- taking medications affecting cognitive function

- alcohol or drug abuse (current or past)

- motor, sensory, vision or hearing dysfunction, which could influence the test's performance

- MS relapse or glucocorticosteroid treatment within the last four weeks.

61 healthy volunteers with no evidence of neurological, psychiatric, or systemic diseases affecting cognitive function were also assessed. The first language of all subjects was Polish.

The demographic characteristics of the groups are set out in Table 1.

The median disease duration in the MS group was 7 years (3-13). The median Expanded Disability Status Scale (EDSS) was 3.5 (2-4.5). There were 45 patients with RRMS, 12 patients with SPMS, and four patients with PPMS. Table 2 sets out the demographic and clinical characteristics of PwMS depending on the course of the disease.

\section{Procedures}

This study was approved by the Bioethical Committee at the Regional Chamber of Physicians and Dentists in Bydgoszcz, Poland (No 39/2017, 19 September 2017). Procedures were performed according to international standards for validation [27]. After informed consent was obtained, a demographic and clinical interview was performed, and subjects completed two self-administered questionnaires: Hospital Anxiety and Depression Scale (HADS) and Modified Fatigue Impact Scale (MFIS). Then the neuropsychological tests of the BICAMS battery were administered in this order: the Symbol Digit Modalities Test (SDMT, oral version), the California Verbal Learning Test (CVLT), and the Brief Visuospatial Memory Test (BVMT-R). Finally, a neurological examination with EDSS assessment was performed by an experienced neurologist. All subjects were examined by the same psychologist and neurologist. The examination of 20 participants with MS was repeated after one to three weeks to assess test-retest reliability. All tests included in the BICAMS battery were repeated.

The Symbol Digit Modalities Test [24] measures information processing speed. The SDMT is composed of nine symbols each in association with a single digit from 1 to 9 . These pairs are explained at the top of the sheet as a key. The working area of the page contains a random sequence of these symbols. In the oral version the subjects have to say the correct digits as quickly as possible. They are scored on how many they get right in $90 \mathrm{sec}$. The instruction of administration was translated into the Polish language. No other adaptation (linguistic or cultural) was required.

The California Verbal Learning Test-II [25] is a tool for measuring verbal memory and learning. The CVLT-II is composed of a list of 16 words in four semantic categories that the subject must learn. During administration, the examiner reads the list aloud at the approximate speed of one word per second. The subject listens to the complete list and is then asked to repeat back as many as possible, in any order, which the examiner records on paper. There is no time limit for this test. The procedure is repeated five times. The score is composed of the total number of words recorded across the five trials. In this study, the Polish adaptation of CVLT [29] was used. The Polish adaptation of CVLT contains the same number of words and number of trials as CVLT-II, and the words are culturally adapted for the Polish population.

The Brief Visuospatial Memory Test - Revised [26] is a test to evaluate visual memory and learning. The BVMT-R consist of six abstract figures on the sheet. This group of figures is shown to the subjects for 10 seconds. After this time, the page is removed and they are asked to draw as many figures as they can remember in the given order on a blank page. Each design is scored from zero to two points, based on accuracy and location criteria. This is repeated three times. The total score is the sum of all three trials. The instruction of administration was translated into the Polish language. No other adaptation (linguistic or cultural) was required. 
Depression and anxiety were measured using the Hospital Anxiety and Depression Scale HADS) [30]. The HADS is a quick self-reported questionnaire containing 14 items, seven questions testing anxiety (HADS-A) and seven testing depression (HADS-D). Each item is rated from 0 to 3, which gives a possible range of scores from 0 (no symptoms) to 21 (most severe symptoms) for each subscale. Scores below 8 are normal. The HADS has been validated in MS [31]. The Polish version of the HADS [32] was used in this study.

The Polish version of the Modified Fatigue Impact Scale (MFIS) was used to measure fatigue [33]. The MFIS is a modified form of the Fatigue Impact Scale [34]. The MFIS contains 21 items, 10 questions relating to mental fatigue, and 11 questions relating to physical and social fatigue. Each item is rated on a scale from 0 (never) to 4 (almost always). The scoring ranges between 0 and 84 , with a high score reflecting a greater impact. A cut-off value of 38 has been used to discriminate fatigued from non-fatigued patients.

\section{Statistical analysis}

Statistical analysis was performed using licensed software (Statistica 13.3, StatSoft). The statistical significance value was set at $\mathrm{p}<0.05$. Normal distribution of data was verified using the Shapiro-Wilk test. The mean (with standard deviation) was used as a measure of central tendency for data with normal distribution. Otherwise, the median (with 25 and 75 percentile) was presented. The comparison between two groups for continuous variables was performed using:

- $\mathrm{t}$-test for independent variables - for variables with normal distribution and homogeneous variance in the groups; the homogeneity of variance was tested using Levene's test,

- $t$-test for independent variables with Welch's correction for variables with normal distribution but unequal variances in the groups,

- Mann-Whitney U test - for variables not meeting the assumption of normality.

The comparison between more than two groups for continuous variables was performed using one-way ANOVA with Tukey's post hoc analysis where it was applicable: otherwise Kruskal-Wallis test was performed. The chi-square test was used to compare two groups for categorial variables. To assess correlation between two variables parametric test (Pearson's correlation) or nonparametric test (Spearman rank correlation) was used depending on the character of a variable's distribution. Correlation of BICAMS test-retest scores was evaluated by the Pearson correlation in accordance with the recommendations. In the analysis regarding more than one predictor of independent variable, a multiple regression model was applied.

\section{Results}

MS patients performed significantly worse on all three tests included in BICAMS compared to healthy subjects (Tab. 3).
Table 3. CVLT, BVMT-R and SDMT results in MS and HC groups

\begin{tabular}{lccc} 
& MS & HC & p \\
& $\mathbf{N}=61$ & $\mathbf{N}=61$ & \\
\hline CVLT, mean (SD) & 51.7 & 56.1 & 0.02 \\
& $(10.9)$ & $(9.2)$ & \\
BVMT-R, median & 25 & 28 & 0.03 \\
(25-75 percentile) & $(19-31)$ & $(24-31)$ & \\
BVMT-R, & 24.0 & 27.1 & - \\
mean (SD) & $(7.7)$ & $(5.7)$ & \\
SDMT, mean (SD) & 48.8 & 57.2 & $<0.001$ \\
& $(12.1)$ & $(9.7)$ &
\end{tabular}

Mann-Whitney U Test was used to assess the differences between the groups for BVMTR, T-Test wa used for CVLT and SDMT. MS - multiple sclerosis, HC - healthy control, CVLT - California Verbal Learning Test, BVMTR — Brief Visuospatial Memory Test-Revised, SDMT — Symbol Digit Modalities Test, significant differences $(p<0.05)$ marked in bold

Table 4. HADS and MFIS scores in MS and HC groups

\begin{tabular}{lccc} 
& $\begin{array}{c}\text { MS } \\
\mathbf{N}=61\end{array}$ & $\begin{array}{c}\text { HC } \\
\mathbf{N}=61\end{array}$ & $\mathbf{p}$ \\
\hline HADS-A, median & 6 & 5 & 0.42 \\
(25-75 percentile) & $(2-9)$ & $(2-7)$ & \\
HADS-D median & 3 & 2 & 0.13 \\
(25-75 percentile) & $(1-6)$ & $(1-4)$ & \\
MFIS total score, median, & 32 & 14 & $<0.001$ \\
(25-75 percentile) & $(19-42)$ & $(6-26)$ & \\
MFIS cognitive functioning, & 12 & 8 & 0.01 \\
median (25-75 percentile) & $(5-19)$ & $(3-13)$ &
\end{tabular}

Mann-Whitney U Test was used to assess the differences between the groups, MS - multiple sclerosis, $\mathrm{HC}$ - healthy control, HADS-A - anxiety subscale of Hospital Anxiety and Depression Scale, HADS-D - depression subscale of Hospital Anxiety and Depression Scale, MFIS - Modified Fatigue Impact Scale, significant differences $(p<0.05)$ marked in bold

Effect sizes (Cohen's d) were: 0.43 for CVLT, 0.46 for BVMT-R and 0.77 for SDMT.

Regarding test-retest reliability, all BICAMS tests produced significant correlations $(r=0.83$ for CVLT, $p<0.001 ; r=0.84$, $\mathrm{p}<0.001$ for BVMTR; $\mathrm{r}=0.9, \mathrm{p}<0.001$ for SDMT).

Table 4 presents the results of the self-administered questionnaires (HADS and MFIS). The depression and anxiety subscales of the HADS were analysed separately. Significant anxiety symptoms were reported by 19 MS patients (31.1\%) and 15 healthy subjects (24.6\%). Significant depressive symptoms were reported by $11 \mathrm{MS}$ patients (18\%) and five healthy volunteers $(8.2 \%)$. Using a cut-off point of 38 for the MFIS, 19 MS patients (31.1\%) and eight HC subjects (13.1\%) reported significant fatigue. MFIS scores and subscale for cognitive aspect of fatigue were considered. Significant differences between the MS and HC groups were noted regarding the MFIS total score and MFIS - cognitive functioning. There was no difference in the case of the HADS (neither in depression nor anxiety symptoms).

In the MS group, we found correlations between age and both BVMT-R and SDMT $(\mathrm{R}=-0.26, \mathrm{p}=0.04 ; \mathrm{R}=-0.28$, $\mathrm{p}=0.03$ respectively), as well as between education and BICAMS tests (CVLT R $=0.42, \mathrm{p}<0.001$, BVMT-R R $=0.5, \mathrm{p}<$ 0.001$, SDMT R $=0.36, \mathrm{p}=0.005)$. Similarly, associations were 
noticed in the HC group in the case of education $(\mathrm{R}=0.47$, $\mathrm{p}<0.001, \mathrm{R}=0.27, \mathrm{p}=0.04, \mathrm{R}=0.44, \mathrm{p}<0.001$ respectively). However, in the HC group, age was correlated only with SDMT $(\mathrm{R}=-0.35, \mathrm{p}=0.005)$. No association of BICAMS to gender was observed. Unemployed PwMS performed worse than employed subjects on the CVLT (unemployed 42.7, SD 9.9; employed 53.5, SD 10.3; $\mathrm{p}=0.003$ ) as well as on the SDMT (unemployed 41.9, SD 12.4; employed 50.2, SD 11.7; $\mathrm{p}=0.04)$. There was no relationship of employment status to BVMT-R.

When considering the differences of cognitive performance associated with the clinical courses of MS, we excluded the PPMS group because of the small number of patients. Significant differences in performance on all three tests were observed between the SPMS and HC groups ( $p=0.02$ for CVLT, $\mathrm{p}=0.02$ for BVM-TR, $\mathrm{p}<0.001$ for SDMT). Moreover, a significant difference between SPMS and RRMS for SDMT $(\mathrm{p}=0.01)$ was also found (Tab. 5).

There was no significant association of the BICAMS test with either HADS or MFIS scores.

Patients with higher EDSS performed worse on CVLT, BVMT-R and SDMT $(\mathrm{R}=-0.31, \mathrm{p}=0.01 ; \mathrm{R}=-0.27, \mathrm{p}=0.04$; $\mathrm{R}=-0.58, \mathrm{p}<0.001$ respectively). BVMT-R and SDMT results were also negatively correlated with duration of the disease $(\mathrm{R}$ $=-0.26, \mathrm{p}=0.04 ; \mathrm{R}=-0.32, \mathrm{p}=0.01$ ).

We also analysed BICAMS scores within the MS group in a regression model including age, education, EDSS and duration of the disease (Tab. 6). This analysis revealed that EDSS was the only predictor of SDMT, whereas education was a predictor of BVMTR and CVLT scores.

Table 5. BICAMS scores for MS and HC subgroups

\begin{tabular}{|lccc|} 
& $\begin{array}{c}\text { RRMS } \\
\text { N }=\mathbf{4 5}\end{array}$ & $\begin{array}{c}\text { SPMS } \\
\mathbf{N}=\mathbf{1 2}\end{array}$ & $\begin{array}{c}\text { HC } \\
\mathbf{N}=\mathbf{6 1}\end{array}$ \\
\hline CVLT, mean (SD) & 54.6 & 45.5 & 56.1 \\
& $(9.7)$ & $(9.5)$ & $(9.2)$ \\
BVMT-R, median & 25 & 21 & 28 \\
(25-75 percentile) & $(20-32)$ & $(16-26)$ & $(24-31)$ \\
SDMT, median & 53 & 43 & 56 \\
(25-75 percentile) & $(46-60)$ & $(30-47)$ & $(52-65)$ \\
\hline
\end{tabular}

RRMS - relapsing-remitting multiple sclerosis, SPMS - secondary progressive multiple sclerosis, HC - healthy control, CVLT - California Verbal Learning Test, BVMTR - Brief Visuospatial Memory Test-Revised, SDMT — Symbol Digit Modalities Test
We identified the $5^{\text {th }}$ percentile value in the HC group for each component of BICAMS to estimate how many MS patients underperformed (Tab. 7). We identified $21 \mathrm{MS}$ patients (34\%) presenting with cognitive dysfunction based on the criterion of one or more test result below the $5^{\text {th }}$ percentile value of the HC group. $11 \mathrm{MS}$ patients (18\%) performed poorly on only one BICAMS test, six patients (10\%) performed poorly on two BICAMS components, while four MS subjects (7\%) demonstrated impairment on all three tests.

\section{Discussion}

Evidence regarding the prevalence of CI in MS has increased significantly in recent years. Results of studies on CI in MS have raised awareness of the seriousness of this problem, particularly in relation to its negative impact on the daily lives of MS patients. CI results in a limitation of social and interpersonal activity, as well as of the ability to work and/ or study and of treatment adherence and rehabilitation [23].

Following these findings, some tools aimed at CI assessment in MS patients were developed, but a comprehensive clinical cognitive assessment requires additional expertise, which is not available in many MS centres, particularly smaller ones. This resulted in the need for a short cognitive testing battery which captures cognitive performance of MS patients and can be quickly and efficiently applied in everyday practice by neurologists. An expert consensus committee of neurologists and neuropsychologists developed the BICAMS battery, offering adequate reliability, sensitivity, specificity, and repeatability [23]. Based on validation standards recommended by an expert panel [27], BICAMS has been successfully validated, and it is currently used in many countries [35].

Table 7. Cognitive impairment in MS patients according to the 5th percentile values of $\mathrm{HC}$ group

\begin{tabular}{lcc} 
& $\begin{array}{c}5^{\text {th }} \text { percentile value } \\
\text { of HC }\end{array}$ & $\begin{array}{c}\text { Number (\%) of MS } \\
\text { patients with lower } \\
\text { score }\end{array}$ \\
\hline CVLT & 41 & $8(13 \%)$ \\
BVMTR & 18 & $12(20 \%)$ \\
SDMT & 40 & $15(25 \%)$
\end{tabular}

HC - healthy control, MS - multiple sclerosis, CVLT — California Verbal Learning Test, BVMTR Brief Visuospatial Memory Test-Revised, SDMT — Symbol Digit Modalities Test

Table 6. Associations between clinical factors and BICAMS scores - multiple regression analysis

\begin{tabular}{|c|c|c|c|c|c|c|}
\hline & \multicolumn{2}{|c|}{ CVLT } & \multicolumn{2}{|c|}{ BVMTR } & \multicolumn{2}{|c|}{ SDMT } \\
\hline & Standardised B & p & Standardised B & $\mathbf{p}$ & Standardised B & $\mathbf{p}$ \\
\hline Age & 0.003 & 0.98 & -0.20 & 0.13 & -0.06 & 0.65 \\
\hline Education & 0.35 & 0.006 & 0.42 & $<0.001$ & 0.17 & 0.13 \\
\hline EDSS & -0.14 & 0.34 & -0.05 & 0.71 & -0.51 & $<0.001$ \\
\hline Duration of the disease & -0.17 & 0.23 & -0.12 & 0.36 & -0.03 & 0.82 \\
\hline
\end{tabular}

CVLT — California Verbal Learning Test, BVMTR - Brief Visuospatial Memory Test-Revised, SDMT - Symbol Digit Modalities Test, EDSS - Expanded Disability Status Scale, significant correlations ( $p<0.05$ ) marked in bold 
We found a significant difference in scores between PwMS and HC. This was most pronounced for the SDMT, where scores for MS patients were substantially lower compared to HC. However, our findings are consistent with the results of earlier studies [36-39]. When using a criterion of at least one test below the normal $\mathrm{HC}$ range, we found cognitive deficits in $34 \%$ of MS patients. We also demonstrated a very strong correlation in test-retest reliability assessment, which is also concordant with earlier data. In our study, there was a significant difference in employment levels between the MS and HC groups. This is a well-established phenomenon in previous BICAMS validation studies, and it is known that unemployment is high for people with MS, even with low EDSS, both in Poland [40] and across Europe [41]. The much higher unemployment rate of the MS group reflects the reality of MS, and we do not consider this to be a statistical anomaly.

However, the prevalence of $\mathrm{CI}$ was lower in our population than in other recent studies [36-38, 42], which is probably a result of the short duration of the disease in the majority of our RRMS patients. A recent BICAMS validation with a short mean disease duration reported similar impairment levels [39]. This can be confirmed by the effect of the disease phenotype on test results, with RRMS patients (with the shortest duration of the disease) receiving the best scores, and SPMS patients (with the longest duration of the disease) the worst. The difference between RRMS and SPMS was significant for SDMT. Scores of SPMS patients and HC differed significantly for all three tests. Our findings confirm earlier findings describing different patterns of $\mathrm{CI}$ in different MS phenotypes and in relation to the duration of the disease $[5,10]$.

Age-related cognitive decline has been well documented in the scientific literature, in terms of information processing speed, verbal memory and visuospatial abilities [43]. A negative correlation of test scores in the BICAMS battery with age, both in HC and PwMS, is therefore to be expected. Our data has confirmed previous results regarding the correlation of age with SDMT and BVMT-R in PwMS and with SDMT in the $\mathrm{HC}$ group.

Education was associated with higher cognitive test scores, possibly as an indicator of cognitive reserve. We did not find a difference in years of education between HC and PwMS. A correlation of CVLT and SDMT with the level of education has been previously reported $[29,44]$. The BVMT-R manual states that education does not influence performance [26], and in another normative study the level of education correlated only with CVLT performance [45]. In this study, we described the significant effect of the level of education on all BICAMS tests, both in MS patients and HC. Some previous data indicates more severe cognitive decline in male PwMS compared to females [46], which can lead to even more pronounced differences between genders for tests in which male subjects routinely perform worse (e.g. CLVT). However, in our study, the BICAMS test results were not associated with gender. We found that CVLT was a strong predictor for occupational activity in PwMS, which is similar to the observation made in the Czech validation of BICAMS and MACFIMS scales [36]. But we also found a similar relationship for SDMT.

Our study confirms earlier findings of a correlation of cognitive decline with motor disability, as assessed by EDSS, and the duration of the disease. Longer duration of the disease and a higher EDSS score have been significantly correlated with CI severity in MS patients [5]. Both those parameters are related, as EDSS scores strongly correlate with the duration of the disease. However, in our dataset we established EDSS as the strongest predictor of SDMT performance using a multiple regression model. In this model, we observed that education was the strongest predictor of CVLT and BVMTR, while the effect of EDSS and duration of the disease became insignificant for these BICAMS components. The protective effect of a high level of education on cognitive deterioration in patients with MS was noted in previous studies [47]. Moreover, CVLT performance is associated with education in healthy subjects. In this study, education also correlated with all the BICAMS components in the $\mathrm{HC}$ group. While our sample of MS patients had low mean EDSS level and relatively short duration of the disease, it is interesting that the variance accounted for by education exceeded that of both EDSS and duration of disease.

Previous validation and other studies using BICAMS have investigated the relation of clinical and demographic variables to BICAMS performance. Mood disorders were observed in the form of depression in $18 \%$ and $8.2 \%$, and as anxiety in $31.1 \%$ and $24.6 \%$ of MS patients and HC, respectively. The difference in prevalence between MS and HC was not significant. However, both prevalence and severity of depression were notably lower in our group compared to earlier studies, in which clinically meaningful depression was observed in $30-50 \%$ of patients [11-12]. A link between depressive symptoms and verbal memory performance has been described [19] and the influence of depression on processing speed is also known [48], but in our study we found no correlation between the HADS depression subscale and BICAMS tests results.

The association between the intensity of anxiety symptoms and cognitive impairment in MS patients is weaker than for depressive symptoms, as has been suggested previously [17]. We found no correlation between the intensity of anxiety symptoms and the extent of cognitive impairment. Data regarding the impact of affective symptoms on cognition is not uniform; the lack of such influence was described in a recent study [49], but the influence of other factors (such as EDSS, disease duration and fatigue) on cognitive performance was emphasised.

As expected, fatigue was more prevalent in MS patients compared to controls, both when assessed by the overall MFIS and the MFIS-cognitive functioning subscale. Approximately $30 \%$ of our MS patients reported significant symptoms of fatigue. An association between fatigue and cognitive impairment has been shown in some studies, especially in tasks requiring prolonged attention [19]. This was confirmed by the 
Hungarian validation work [37], where a strong correlation of fatigue with performance on all three BICAMS tests was observed. Nevertheless, a recent study on a large group of patients did not confirm the effect of fatigue on processing speed, attention, executive function, and memory [50], which is consistent with the results of our study.

\section{Conclusions}

The Polish version of BICAMS is a sensitive, specific, and short battery of cognitive tests, allowing the assessment and monitoring of cognitive impairment in MS patients.

\section{References}

1. Compston A, Coles A. Multiple sclerosis. The Lancet. 2002; 359(9313): 1221-1231, doi: 10.1016/s0140-6736(02)08220-x.

2. Chiaravalloti N, DeLuca J. Cognitive impairment in multiple sclerosis. The Lancet Neurology. 2008; 7(12): 1139-1151, doi: 10.1016/ s1474-4422(08)70259-x.

3. Langdon D. Cognition in multiple sclerosis. Current Opinion in Neurology. 2011; 24(3): 244-249, doi: 10.1097/wco.0b013e328346a43b.

4. Benedict R, Wahlig E, Bakshi R, et al. Predicting quality of life in multiple sclerosis: accounting for physical disability, fatigue, cognition, mood disorder, personality, and behavior change. Journal of the Neurological Sciences. 2005; 231(1-2): 29-34, doi: 10.1016/j.jns.2004.12.009.

5. Amato M, Portaccio E, Goretti B, et al. Cognitive impairment in early stages of multiple sclerosis. Neurological Sciences. 2010; 31(S2): 211-214, doi: 10.1007/s10072-010-0376-4.

6. Lebrun C, Blanc F, Brassat D, et al. Cognitive function in radiologically isolated syndrome. Multiple Sclerosis Journal. 2010; 16(8): 919-925, doi: $10.1177 / 1352458510375707$.

7. Zipoli V, Goretti B, Hakiki B, et al. Cognitive impairment predicts conversion to multiple sclerosis in clinically isolated syndromes. Multiple Sclerosis Journal. 2009; 16(1): 62-67, doi: 10.1177/1352458509350311.

8. Deloire M, Ruet A, Hamel D, et al. Early cognitive impairment in multiple sclerosis predicts disability outcome several years later. Multiple Sclerosis Journal. 2010; 16(5): 581-587, doi: 10.1177/1352458510362819.

9. Staff N, Lucchinetti C, Keegan B. Multiple Sclerosis With Predominant, Severe Cognitive Impairment. Archives of Neurology. 2009; 66(9), doi: 10.1001/archneurol.2009.190.

10. BENEDICT R, COOKFAIR D, GAVETT R, et al. Validity of the minimal assessment of cognitive function in multiple sclerosis (MACFIMS). Journal of the International Neuropsychological Society. 2006; 12(04), doi: 10.1017/s1355617706060723.

11. Siegert RJ. Depression in multiple sclerosis: a review. Journal of Neurology, Neurosurgery \& Psychiatry. 2005; 76(4): 469-475, doi: 10.1136/jnnp.2004.054635.

12. Beiske AG, Svensson E, Sandanger I, et al. Depression and anxiety amongst multiple sclerosis patients. European Journal of Neurology. 2008; 15(3): 239-245, doi: 10.1111/j.1468-1331.2007.02041.x.

13. Benedict R, Cox D, Thompson L, et al. Reliable screening for neuropsychological impairment in multiple sclerosis. Multiple Sclerosis Journal. 2016; 10(6): 675-678, doi: 10.1191/1352458504ms1098oa.

14. Julian L, Merluzzi NM, Mohr DC. The relationship among depression, subjective cognitive impairment, and neuropsychological performance in multiple sclerosis. Multiple Sclerosis Journal. 2016; 13(1): 81-86, doi: $10.1177 / 1352458506070255$.

15. Kinsinger S, Lattie E, Mohr D. Relationship between depression, fatigue, subjective cognitive impairment, and objective neuropsychological functioning in patients with multiple sclerosis. Neuropsychology. 2010; 24(5): 573-580, doi: 10.1037/a0019222.

16. ARNETT P, BARWICK F, BEENEY J. Depression in multiple sclerosis: Review and theoretical proposal. Journal of the International Neuropsychological Society. 2008; 14(5): 691-724, doi: 10.1017/ s1355617708081174.

17. Simioni S, Ruffieux C, Bruggimann L, et al. Cognition, mood and fatigue in patients in the early stage of multiple sclerosis. Swiss Med Wkly. 2007; 137(35-36): 496-501, doi: 2007/35/smw-11874, indexed in Pubmed. ; 17990136.

18. Minden SL, Frankel D, Hadden L, et al. The Sonya Slifka Longitudinal Multiple Sclerosis Study: methods and sample characteristics. Multiple Sclerosis Journal. 2016; 12(1): 24-38, doi: 10.1191/135248506ms12620a.

19. Heesen C, Schulz KH, Fiehler J, et al. Correlates of cognitive dysfunction in multiple sclerosis. Brain, Behavior, and Immunity. 2010; 24(7): 1148-1155, doi: 10.1016/j.bbi.2010.05.006.

20. Bol Y, Duits A, Hupperts R, et al. The impact of fatigue on cognitive functioning in patients with multiple sclerosis. Clinical Rehabilitation. 2010; 24(9): 854-862, doi: 10.1177/0269215510367540.

21. Rao SMA. manual for the Brief, Repeatable Battery of Neuropsychological Tests in Multiple Sclerosis. New York: National Multiple Sclerosis Society. ; 1991.

22. Benedict R, Fischer J, Archibald C, et al. Minimal Neuropsychological Assessment of MS Patients: A Consensus Approach. The Clinical Neuropsychologist. 2010; 16(3): 381-397, doi: 10.1076/ clin.16.3.381.13859.

23. Langdon DW, Amato MP, Boringa J, et al. Recommendations for a Brief International Cognitive Assessment for Multiple Sclerosis (BICAMS). Multiple Sclerosis Journal. 2011; 18(6): 891-898, doi: 10.1177/1352458511431076.

24. Smith A. Symbol digit modalities test: Manual. Los Angeles: Western Psychological Services. ; 1982.

25. Delis D, Kramer J, Kaplan E, et al. California Verbal Learning Test--Second Edition. PsycTESTS Dataset. 2016, doi: 10.1037/t15072-000.

26. Benedict RH. Brief Visuospatial Memory Test - Revised: Professional Manual. FPAR, Inc. , Odessa. ; 1997.

27. Benedict R, Amato M, Boringa J, et al. Brief International Cognitive Assessment for MS (BICAMS): international standards for validation. BMC Neurology. 2012; 12(1), doi: 10.1186/1471-2377-12-55.

28. Polman $\mathrm{CH}$, Reingold SC, Banwell B, et al. Diagnostic criteria for multiple sclerosis: 2010 revisions to the McDonald criteria. Ann Neurol. 2011; 69(2): 292-302, doi: 10.1002/ana.22366, indexed in Pubmed: 21387374.

29. Łojek E, Stańczak J. Podręcznik do Kalifornijskiego Testu Uczenia się Językowego Deana C. Delisa, Joela H. Kramera, Edith Kaplan i Beth A. Ober. Pracownia Testów Psychologicznych Polskiego Towarzystwa Psychologicznego. Warszawa. ; 2012.

30. Zigmond AS, Snaith RP. The Hospital Anxiety and Depression Scale. Acta Psychiatrica Scandinavica. 1983; 67(6): 361-370, doi: 10.1111/j.1600-0447.1983.tb09716.x.

31. Honarmand K, Feinstein A. Validation of the Hospital Anxiety and Depression Scale for use with multiple sclerosis patients. Mult Scler. 2009; 15(12): 1518-1524, doi: 10.1177/1352458509347150, indexed in Pubmed: 19965520. 
32. Karakuła H, Grzywa A, et al. Śpila B, Zastosowanie Skali Lęku i Depresji - HADS w chorobach psychosomatycznych. Psychiatr Pol. 1996; 4: 653-668.

33. Gruszczak A, Bartosik-Psujek H, et al. Pocińska K, 2009. Analiza walidacyjna wybranych aspektów psychometrycznych polskiej wersji Zmodyfikowanej Skali Wpływu Zmęczenia (Modifed Fatigue Impact Scale) - wyniki wstępne. Neurol Neurochir Pol. 2009; 43(2): 148154, indexed in Pubmed. ; 19484692.

34. Fisk JD, Pontefract A, Ritvo PG, et al. The impact of fatigue on patients with multiple sclerosis. Can J Neurol Sci. 1994; 21(1): 9-14, indexed in Pubmed: 8180914.

35. Corfield F, Langdon D. A Systematic Review and Meta-Analysis of the Brief Cognitive Assessment for Multiple Sclerosis (BICAMS). Neurol Ther. 2018; 7(2): 287-306, doi: 10.1007/s40120-018-0102-3, indexed in Pubmed: 29923070.

36. Dusankova J, Kalincik T, Havrdova E, et al. Cross Cultural Validation of The Minimal Assessment of Cognitive Function in Multiple Sclerosis (MACFIMS) and The Brief International Cognitive Assessment for Multiple Sclerosis (BICAMS). The Clinical Neuropsychologist. 2012; 26(7): 1186-1200, doi: 10.1080/13854046.2012.725101.

37. Sandi D, Rudisch T, Füvesi J, et al. The Hungarian validation of the Brief International Cognitive Assessment for Multiple Sclerosis (BICAMS) battery and the correlation of cognitive impairment with fatigue and quality of life. Mult Scler Relat Disord. 2015; 4(6): 499-504, doi: 10.1016/j.msard.2015.07.006, indexed in Pubmed: 26590654.

38. Polychroniadou E, Bakirtzis C, Langdon D, et al. Validation of the Brief International Cognitive Assessment for Multiple Sclerosis (BICAMS) in Greek population with multiple sclerosis. Multiple Sclerosis and Related Disorders. 2016; 9: 68-72, doi: 10.1016/j.msard.2016.06.011.

39. Sousa C, Rigueiro-Neves M, Miranda T, et al. Validation of the brief international cognitive assessment for multiple sclerosis (BICAMS) in the Portuguese population with multiple sclerosis. BMC Neurol. 2018; 18(1): 172, doi: 10.1186/s12883-018-1175-4, indexed in Pubmed: 30332999.

40. Selmaj K, Kobelt G, Berg J, et al. European Multiple Sclerosis Platform. New insights into the burden and costs of multiple sclerosis in Europe: Results for Poland. Mult Scler. 2017; 23(2_suppl): 130-142, doi: 10.1177/1352458517708666, indexed in Pubmed: 28643586.
41. Kobelt G, Thompson A, Berg J, et al. MSCOI Study Group, European Multiple Sclerosis Platform. New insights into the burden and costs of multiple sclerosis in Europe. Mult Scler. 2017; 23(8): 1123-1136, doi: 10.1177/1352458517694432, indexed in Pubmed: 28273775.

42. O'Connell K, Langdon D, Tubridy N, et al. A preliminary validation of the brief international cognitive assessment for multiple sclerosis (BICAMS) tool in an Irish population with multiple sclerosis (MS). Mult Scler Relat Disord. 2015; 4(6): 521-525, doi: 10.1016/j. msard.2015.07.012, indexed in Pubmed: 26590658.

43. Harada C, Love MN, Triebel K. Normal Cognitive Aging. Clinics in Geriatric Medicine. 2013; 29(4): 737-752, doi: 10.1016/j. cger.2013.07.002.

44. SHERIDAN L, FITZGERALD H, ADAMS K, et al. Normative Symbol Digit Modalities Test performance in a community-based sample. Archives of Clinical Neuropsychology. 2006; 21(1): 23-28, doi: 10.1016/j. acn.2005.07.003.

45. Goretti B, Niccolai C, Hakiki B, et al. The brief international cognitive assessment for multiple sclerosis (BICAMS): normative values with gender, age and education corrections in the Italian population. BMC Neurology. 2014; 14(1), doi: 10.1186/s12883-014-0171-6.

46. Beatty W, Aupperle R. Sex Differences in Cognitive Impairment in Multiple Sclerosis. The Clinical Neuropsychologist. 2010; 16(4): 472-480, doi: 10.1076/clin.16.4.472.13904.

47. Martins Da Silva A, Cavaco S, Moreira I, et al. Cognitive reserve in multiple sclerosis: Protective effects of education. Mult Scler. 2015; 21(10): 1312-1321, doi: 10.1177/1352458515581874, indexed in Pubmed: 25948624.

48. Lubrini G, Lago MR, Periañez J, et al. The contribution of depressive symptoms to slowness of information processing in relapsing remitting multiple sclerosis. Multiple Sclerosis Journal. 2016; 22(12): 1607-1615, doi: 10.1177/1352458516661047.

49. Karadayi H, Arisoy O, Altunrende B, et al. The relationship of cognitive impairment with neurological and psychiatric variables in multiple sclerosis patients. Int J Psychiatry Clin Pract. 2014; 18(1): 45-51, doi: 10.3109/13651501.2013.845221, indexed in Pubmed: 24047424.

50. Golan D, Doniger G, Wissemann K, et al. The impact of subjective cognitive fatigue and depression on cognitive function in patients with multiple sclerosis. Multiple Sclerosis Journal. 2017; 24(2): 196-204, doi: $10.1177 / 1352458517695470$. 